\title{
Transtorno factício: um desafio para as diversas especialidades
}

\section{Factitious disease: a challenge to several specialities}

\author{
Gustavo Fábio Aranha1', Luiza Zonzini Máximo de Carvalho², \\ Francisco Bevilacqua Guarniero ${ }^{3}$, Simone M. de S. Rita Soares ${ }^{3}$
}

Aranha GF, Carvalho LZM, Guarniero FB, Soares SMSR. Transtorno factício: um desafio para as diversas especialidades. Rev Med (São Paulo). 2007 jan.-mar.;86(1):14-9.

\begin{abstract}
RESUMO: O transtorno factício caracteriza-se por produção de sintomas por parte do paciente de forma intencional, com o intuito de assumir o papel de paciente. É uma doença de graves implicações clínicas e que gera elevados gastos para o sistema de saúde público. O caso da paciente DLK, ilustrado nesse relato, exemplifica bem esse transtorno. A paciente iniciou suas queixas clínicas com alucinações visuais e um quadro delirante. Com o tempo surgiram queixas de dores abdominais, retenção urinária, fraqueza muscular e um quadro psicótico de difícil resolução. A paciente passou por diversas internações e especialistas e foi submetida a diversos exames e uma laparoscopia, todos de resultados frustros. Apresentou melhora progressiva e importante dos sintomas após o estabelecimento de um vínculo positivo com a equipe e redução das medicações utilizadas. A detecção precoce dessa doença é fundamental, reduzindo a morbidade do transtorno e a prática de medidas iatrogênicas que esses pacientes demandam.
\end{abstract}

DESCRITORES: Transtornos psicóticos/diagnóstico. Transtornos psicóticos/psicologia. Transtornos somatoformes/diagnóstico. Transtornos somatoformes/psicologia. Diagnóstico diferencial.

Trabalho premiado no COMU - Área: Relato de Caso

1. Aluno do sexto ano da graduação da FMUSP.

2. Aluna do quinto ano da graduação da FMUSP.

3. Residentes do segundo ano de Psiquiatria da FMUSP.

Endereço para correspondência: Gustavo Fábio Aranha. R. Mateus Grou, 539, ap. 92, Pinheiros - São Paulo, CEP: 05415-050. e-mail: gfaranha@gmail.com 


\section{Relato de Caso}

$\mathrm{D}$ L.K., sexo feminino, 29 anos, solteira, descendência oriental (Japão), natural e procedente de São Paulo, segundo grau completo (iniciou um curso de instrumentação cirúrgica porém não chegou a concluí-lo) anos"

QD: "vejo o diabo no espelho desde os 14

HPMA: paciente refere que aos 14 anos fez um pacto com o diabo para que esse desse vida longa a sua família e em troca ela deveria se suicidar. Desde então refere ver o diabo em qualquer espelho. Ouve vozes ordenando que se mate e que engula objetos metálicos, o que fez algumas vezes. Bate a cabeça na parede pois sente uma vontade incontrolável. Queixa-se de medo das pessoas, por isso olha para o chão continuamente mantendo o cabelo encobrindo o rosto. Diz estar na Lua e não poder voltar para a Terra.

\section{ISDA:}

- cabeça e pescoço: cefaléia diária de forte intensidade, inespecífica desde os 18 anos.

- pulmonar: ndn

- cardiovascular: ndn

- digestório: vômitos diários há aproximadamente dois meses, sem relato de náuseas precedendo. Dor abdominal de forte intensidade, diária, inespecífica

- genito-urinário: há aproximadamente quatro meses apresentava uma retenção urinária sem causa definida, sendo necessário o uso de sonda vesical de demora nesse período.

- músculo-esquelético: fraqueza muscular nos membros inferiores, levando a paciente a usar cadeira de rodas. Tremores de extremidades contínuos de amplitude média

- pele e anexos: ndn

AP:

DNPM normal.

Mãe refere que a paciente sempre foi uma criança isolada, com dificuldade de relacionamento com outras crianças, sendo chamada de estranha e burra. Tinha dificuldades escolares, chegando a repetir dois anos, no entanto conseguiu completar o segundo grau.

Refere crises convulsivas com investigação eletroencefalográfica normal até o momento.

Teve diversas tentativas de suicídio (cortou os pulsos, ingeriu medicações, tentou pular do $13^{\circ}$ andar mas foi pega pelo pai) e geralmente ficava internada na Santa Casa de São Paulo após.

Teve 19 internações psiquiátricas em hospitais diversos. Sua primeira internação ocorreu aos 18 anos porque ingeriu objetos metálicos. Relata uma crise convulsiva prévia que não voltou a se repetir. Refere ter sido expulsa da Santa Casa e passou por atendimento em diversos locais. Relata ter recebido 90 sessões de eletroconvulsoterapia (ECT) ao todo. Há três anos em acompanhamento particular no Hospital Nipo-Brasileiro.

Deu entrada no IPQ - FMUSP fazendo uso de

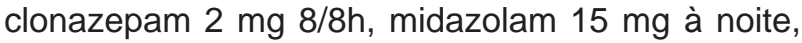
cloridrato de oxibutinina um comprimido/dia, cloxazolam $2 \mathrm{mg}$ à noite, Tylex 30mg 6/6h, Tramal

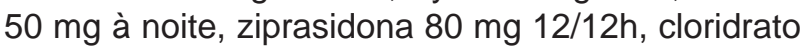

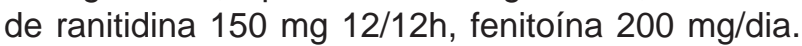
Outras medicações que fez uso: sertralina, paroxetina, fluoxetina, escitalopram, Neuleptil $4 \%$, Neozine, diabiperideno, clorpromazina, Diazepam, alprazolam, olanzapina, carbamazepina, oxcarbazepina, carbonato de lítio, gabapentina, haloperidol, além de diversos anti-inflamatórios, antibióticos e procinéticos.

Foi submetida a extensa investigação laboratorial e de imagem devido às queixas de cefaléia persistente, dor abdominal e crises convulsivas, incluindo radiografias de todo o corpo, ultrassonografia abdominal, endoscopia digestiva alta, eletroencefalogramas (quatro), tomografia de crânio (assimetria de crânio), ressonância nuclear magnética de crânio, sem alterações significativas. Em 2003 chegou a ser submetida a uma laparoscopia para investigação da dor abdominal quando foi retirado um cisto ovariano benigno. A dor persistiu com as mesmas características após a cirurgia.

Em fevereiro de 2005 iniciou quadro de retenção urinária sendo submetida a sondagem vesical. Foi feita tentativa de retirada da sonda, no entanto o quadro persistiu e foi optado pela sondagem vesical de demora, a qual persistia no início da internação no Instituto de Psiquiatria do HC-FMUSP, em junho de 2005. A investigação etiológica não chegou a ser completada.

Nega uso de álcool, drogas ilícitas ou tabagismo. Relata eventualmente cheirar água sanitária e acetona.

AF:

Pais saudáveis.

D. é a segunda filha de uma prole de dois. Irmão saudável, bem sucedido profissionalmente.

Sem outros antecedentes mórbidos psiquiátricos na família.

\section{Contexto familiar}

A paciente e sua mãe apresentam o pai como um homem perturbado, muito queixoso e agressivo 
verbalmente. D. e sua mãe apresentam uma relação de estreita dependência, tendo idéias e discursos muito semelhantes. A mãe da paciente acredita e reforça todos os sintomas da filha, chegando a confrontar agressivamente a equipe médica quando não era dada a medicação esperada para $D$.. O irmão da paciente tem pouco contato com a família, apesar de morar em casa. Isolou-se no estudo e pouco parece compreender sobre a doença da irmã e a dinâmica familiar.

\section{Exame Psíquico (admissão)}

Apresentação descuidada, roupas em mau estado de conservação, higiene prejudicada, tremores de extremidade que tornavam-se mais grosseiros quando ficava angustiada, em uso de sonda vesical de demora. Permanecia a maior parte do tempo deitada no leito, ao sentar-se o fazia na cadeira de rodas e permanecia com a cabeça pendendo para a frente com seus cabelos cobrindo o rosto. Nível de consciência e orientação têmporoespacial preservados, atenção voluntária pouco diminuída, sem alterações de atenção espontânea, humor tendendo para depressivo, intensa labilidade afetiva, sem alteração do tônus e ressonância afetivos. Pensamento de conteúdo delirante (fez um pacto com o diabo e teria que cumpri-lo, sentia-se perseguida por todas pessoas que a cercavam), alucinações auditivas (diabo ordenando que se matasse) e visuais (diabo). Discurso de velocidade normal, coerente, de conteúdo delirante, aparentando ansiedade para expressar-se, em especial sobre suas doenças e delírios. Crítica bastante prejudicada, inteligência aparentemente limítrofe (devido a atitude pueril), porém com um conhecimento detalhado sobre todas as hipóteses diagnósticas já aventadas e as medicações que fazia uso, bem como seus efeitos terapêuticos e colaterais. refratária

HD (entrada): Esquizofrenia paranóide

\section{Evolução}

- entrada: apresentava episódios de agitação psicomotora, acalmando-se após conversa;

- 04/06/2005: iniciada introdução de ácido valpróico (foi aumentado progressivamente até a dose de 1000 mg/dia em 12/06) e redução da fenitoína e analgésicos.

- 07/06/2005: iniciada redução da ziprasidona. Enfermagem observa paciente auto-induzindo vômitos;

- 08/06/2005: já aceita prender o cabelo. Muito insistente na solicitação de medicações;
- 12/06/2005: engole um clipe metálico (diz que foi ordenada pelo diabo);

- 15/06/2005: melhor aceitação da redução de medicamentos. Melhora do contato e disposição;

- 17/06/2005: introduzida sertralina e iniciada redução lenta dos benzodiazepínicos;

- 20/06/2005: retirada sonda vesical de demora. Paciente referiu não conseguir urinar, porém foi vista urinando pela enfermagem duas vezes;

- 24/06/2005: melhora do auto-cuidado (vestese bem, maqueia-se);

- 04/07/2005: queixa-se de sentir-se perseguida (sem saber por quem) e ouvir vozes femininas solicitando que machucasse outras pessoas. Nos dias seguintes teve piora do quadro com novas crises de agitação psicomotora (chegando a ser contida fisicamente em 05/07) e intensa solicitação por medicação;

- 06/07/2005: introduzido risperidona;

-11/07/2005: inicia acompanhamento psicoterápico. Tentativa pueril de suicídio (enrola lençol no pescoço e anda em frente ao posto de enfermagem);

- 13/07/2005: melhora do comportamento e disposição. Inicia atendimento no hospital-dia;

-28/07/2005: após período estável, com eventuais queixas somáticas e de senso-percepção, recebe alta para seguimento em hospital-dia;

- hospital-dia: certa dificuldade de adaptação inicialmente. Muito calada nos grupos, sempre com a cabeça baixa, queixando-se de delírios, porém com pouca freqüência. Teve posterior piora dos delírios, com melhora após introdução de clozapina. No momento em uso de clozapina $400 \mathrm{mg} / \mathrm{dia}$, sertralina $200 \mathrm{mg} / \mathrm{dia}$, ziprasidona $160 \mathrm{mg} / \mathrm{dia}$, oxcarbazepina $900 \mathrm{mg} / \mathrm{dia}$ e propranolol $40 \mathrm{mg} / \mathrm{dia}$ (tremores de extremidades). O quadro alucinatório teve nova piora após algum tempo de uso da clozapina e, no momento queixa-se de ver gatos e cachorros vermelhos que a perseguem. Voltou a queixar-se também de dores abdominais.

\section{Dados complementares}

- Durante a internação foi feito teste neuropsicológico, sugerindo uma depressão psicótica severa. A testagem cognitiva concluiu que a paciente não se encontrava em condições de atender a exigências que demandassem certa organização dos recursos cognitivos, devido aos delírios. Não foi evidenciado nenhum grau de retardo mental.

- Sempre que havia uma redução na medicação ou uma redução na atenção prestada a paciente esta apresentava uma piora das queixas somáticas e agitação. A piora em sua evolução em 
04/07/2005 seguiu-se a admissão de um novo paciente na enfermaria que estava muito agitado e demandava muita atenção da equipe médica e de enfermagem.

\section{Novas HD feitas durante a internação: \\ Transtorno factício \\ Transtorno psicótico?}

\section{DISCUSSÃO}

D. deu entrada com diversas limitações físicas e psíquicas e uma história médica repleta de investigações clínicas que não resultavam em achado de patologias, com intervenções médicas pouco efetivas. Seu quadro psicótico foi tratado com diversas medicações em doses adequadas e ECT, sempre com respostas modestas ou ausentes.

Durante a internação foi notado que $\mathrm{D}$. tinha grande compreensão sobre suas diversas hipóteses diagnósticas bem como das medicações que fazia uso (incluindo efeitos colaterais), o que contrastava com sua aparente limitação intelectual e atitude pueril. Seu comportamento também evidenciava um forte traço histriônico, pois apresentava, por exemplo, piora nos tremores de extremidades ou no aumento de suas queixas somáticas quando em frente à equipe médica ou de enfermagem. Quando seus delírios eram contestados, muitas vezes D. incluía novos elementos aos delírios (p. ex., disse que era a Lady Di e ao ser confrontada que isso não seria possível pois a mesma já havia falecido disse "eu sou o diabo então").

Ao observar tais comportamentos foi optado por redução de suas medicações, havendo sempre grande resistência inicial por parte de D.. Após argumentação e reasseguramento a paciente concordava com a conduta e apresentava estabilidade do quadro, mesmo com menos medicações. A paciente teve uma evolução muito favorável durante a internação, sendo possível a retirada da sonda vesical, passou a prender os cabelos e voltou a deambular, não batia mais a cabeça contra a parede, cessação completa das queixas de dores abdominais e chegou a fazer vínculos com outros pacientes da enfermaria. No entanto persistiram as queixas de alucinações visuais, porém em menor freqüência do que anteriormente.

No transtorno factício, o paciente intencional e conscientemente produz sinais e sintomas psicológicos e/ou físicos para buscar a atenção médica e de sua equipe através da hospitalização. Apesar de os sinais e sintomas serem produzidos conscientemente, a motivação é inconsciente ${ }^{6,7}$. Seu objetivo é o de assumir o papel de enfermo e dessa maneira conseguir atenção. Mesmo tendo uma percepção pobre sobre a motivação para seus comportamentos, eles continuam agindo dessa maneira².

É comum em pacientes com esse transtorno um histórico de várias passagens por médicos e diversas hospitalizações por motivos que, se não idênticos, muito parecidos. Podem se colocar em situações de alto risco, como infecções recorrentes de pele devido à contaminação por fezes, hipoglicemias inexplicadas e recorrentes em pacientes com acesso à insulina, febre de origem indeterminada ou fazer uso indevido de medicações prescritas ${ }^{11}$. Da mesma forma observa-se uma certa satisfação em realizar exames invasivos e até mutiladores, não sendo incomum a presença de diversas cicatrizes nesses pacientes ${ }^{3}$. D. chegou diversas vezes a solicitar que fossem feitos exames, como colonoscopia, e ao ser-lhe dito que seria submetida a algum exame sempre reagia com demonstrações de evidente satisfação.

A maioria dos pacientes apresenta profissão ou interesse em seguir carreira em área da saúde, têm um quociente de inteligência (QI) considerado normal ou acima da média, o que não condiz com o comportamento muitas vezes infantilizado e embotado do paciente. Esses pacientes são bem esclarecidos sobre as patologias atribuídas a eles e os tratamentos disponíveis. São bem informados quanto aos medicamentos usados, aos efeitos dos mesmos e, muitas vezes, solicitam medicamentos específicos como forma de tratamento para a patologia que eles supõem e referem aos profissionais da saúde ${ }^{3}$.

É difícil caracterizar o que motiva essas pessoas a agirem dessa forma. Acredita-se que no fundo os pacientes estão em busca de algo como aceitação, amor e afeto. E essa necessidade que é possivelmente originada de suas relações com os pais, de alguma forma é transferida aos médicos e sua equipe fazendo com que os mesmos substituam os pais nessa dinâmica. Daí a necessidade quase compulsiva de tratamento médico, passagens por vários serviços e dezenas de internações. É evidente um distúrbio importante da auto-imagem e uma identidade perturbada e pobre ${ }^{3,4}$.

Outra característica de pacientes com esse transtorno é a relutância dos mesmos com a menor possibilidade de tratamento psicológico isolado com a alta hospitalar. Quando confrontados com essas possibilidades intensificam os sinais e sintomas apresentados. Se não conseguem controlar a situação podem procurar escapar, fugir do serviço. $E$ no momento seguinte estarão procurando outro 
serviço na região que consiga satisfazer suas necessidades e carências. Não é raro encontrar pacientes com traços fortemente sedutores e manipuladores, de modo a controlar a situação, mesmo lidando com médicos e profissionais extremamente competentes ${ }^{3}$.

Não há dados precisos sobre a prevalência dessa doença, uma vez que o diagnóstico de certeza é difícil e muitos casos nem são diagnosticados. Além do mais, os pacientes geralmente param de freqüentar o hospital onde estavam sendo acompanhados quando são descobertos ${ }^{5}$. No entanto o transtorno é cada vez mais diagnosticado conforme mais se conhece dessa patologia. Acredita-se que cerca de entre 0,5 e $10 \%$ dos pacientes admitidos em um hospital geral têm, na verdade, transtorno factício. A prevalência é maior entre indivíduos entre 20 e 40 anos, mas também foram descritos casos em crianças e idosos. Quanto à prevalência por sexo, a literatura é discordante ${ }^{1,3,4}$.

Os critérios diagnósticos para caracterizar essa doença, segundo o DSM-IV, são:

A. Produção ou simulação intencional de sinais ou sintomas físicos ou psicológicos;

B. A motivação para o comportamento consiste em assumir o papel de enfermo;

C. Incentivos externos para o comportamento (tais como vantagens econômicas, esquiva de responsabilidades legais ou melhora no bem-estar físico) estão ausentes.

São ainda reconhecidos quatro subtipos de transtorno factício:

1. Com sinais e sintomas predominantemente físicos;

2. Com sinais e sintomas predominantemente psicológicos;

3. Com combinação de sinais e sintomas físicos e psicológicos;

4. Sem outra especificação: não preenchem todos os critérios para transtorno factício, como, p.ex., transtorno factício por procuração (um indivíduo produz sinais em outra pessoa) $)^{1,2}$.

O caso torna-se mais desafiador quando os pacientes misturam doenças reais e imaginárias ${ }^{4}$. $\mathrm{Na}$ literatura são descritos casos isolados de paciente que apresentam sintomas psicóticos, mas tais pacientes geralmente desenvolvem uma psicose autêntica quando seguidos longitudinalmente. É possível que a produção de sintomas psicóticos seja, na realidade, uma tentativa de defesa contra a emergência de um quadro psicótico genuíno ${ }^{10}$. Tal descrição pode aplicar-se ao caso em questão, uma vez que no decorrer do tratamento todos os sintomas da paciente remitiram, permanecendo, no entanto, um núcleo psicótico.
Pode ser difícil distinguir uma psicose verdadeira de uma factícia. Para tal deve-se ter uma observação continuada das condutas e resposta às medicações que esses pacientes apresentam, sendo isso feito preferencialmente com o paciente internado ${ }^{8}$. Esses pacientes podem ter sintomas psicológicos que são facilmente sugestionáveis, o que pode auxiliar no diagnóstico ${ }^{5}$.

O teste neuropsicológico pode fornecer resultados inválidos ou falso-positivos, pois os pacientes tendem a manipular as respostas de maneira a parecerem doentes ${ }^{4}$. $\mathrm{O}$ teste realizado pela paciente sugeriu uma depressão psicótica grave, quadro que não foi evidenciado no contato diário com a paciente.

A melhora das queixas de D. deu-se, em grande parte, devido ao rompimento do ciclo de manipulação e persuasão na relação da paciente com a equipe médica. Quando passou a ser acompanhada ambulatorialmente com outra equipe tentou novamente exercer um domínio na relação médico-paciente, colocando-se no papel de enferma, na busca por afeto e aceitação pela figura do profissional de saúde.

Esse caso é exemplar quanto a mobilização que pacientes com esse transtorno geram, sendo submetidos a diversos diagnósticos e tratamentos. No entanto, essa conduta só leva ao reforço da atuação, prorrogando e agravando ainda mais o quadro, além de representar um grande gasto para a saúde pública. Os custos humanos são significativos, com taxas elevadas de morbimortalidade. Além disso tendem a despertar uma contratransferência negativa na equipe médica quando é evidenciada a natureza factícia da doença, com sentimentos de angústia e raiva ${ }^{2,7}$.

A abordagem recomendada é a de não confrontar o paciente com a falsidade de seus sinais e sintomas, oferecendo, no entanto, medidas que o permitam se recuperar. Eles devem ser informados do plano de tratamento e envolvidos no mesmo. Técnicas comportamentais podem ser empregadas e a psicoterapia auxilia no reforço da identidade e auto-estima. É muito raro que o paciente reconheça perante a equipe a falsidade dos sinais e sintomas ${ }^{1,7}$.

O prognóstico do transtorno factício é pobre, em especial quando há sintomas psicológicos. Pope et al. ${ }^{9}$ estudaram 219 pacientes com quadros psicóticos consecutivamente admitidos em um hospital, dos quais nove (4,1\% do total) apresentavam transtorno factício. Foi realizado um follow-up entre quatro e sete anos e observou-se que um paciente suicidou-se, quatro estavam hospitalizados no momento da avaliação de follow-up e outros três haviam tido uma ou mais hospitalizações 
subseqüentes ao episódio no qual haviam sido admitidos no estudo. Apenas um paciente não teve história de outras hospitalizações. Foi feita ainda uma comparação com pacientes com esquizofrenia, transtorno esquizoafetivo e mania, onde verificou-se que os pacientes com transtorno factício têm um pior prognóstico em todas as funções avaliadas (social, ocupacional, sintomas residuais e funcionamento global).

As diversas queixas somáticas e psíquicas que esses pacientes apresentam fazem com que sejam um desafio para as diversas especialidades médicas.
O diagnóstico é difícil, sendo geralmente necessária a realização de diversos exames e um acompanhamento prolongado até que essa hipótese seja aventada. Paradoxalmente, a estratégia mais importante no manejo desses pacientes é o diagnóstico precoce, prevenindo assim um aumento do sofrimento e do risco associado a auto-lesões e exames desnecessários. Além do mais, em estágios precoces da doença uma intervenção psiquiátrica tende a ser melhor sucedida ${ }^{7}$. É fundamental, portanto, que profissionais de todas especialidades estejam atentos para tal possibilidade.

Aranha GF, Carvalho LZM, Guarniero FB, Soares SMSR. Factitious disease: a challenge to several specialities. Rev Med (São Paulo). 2007 jan.-mar.;86(1):14-9.

\begin{abstract}
In factitious disease there is an intentional production of symptoms by the patient, with the intent of being sick in front of others. It is a disease with serious clinical implications and these patients represent a enormous waste of money to public health. The case of DLK exemplifies it. The complaints of the patient began with delusions and visual hallucinations. She developed abdominal pains, urinary retention, weakness and psychotic symptoms of difficult management. She had gone to several hospitals, submitted to many exams and a laparoscopy, all with frustrating results. There was a progressive and important improvement by the establishment of a positive relation with the patient and reduction of her medications. Early recognition of this disease is fundamental, diminishing the morbidity of this disease and the practice of iatrogenic measures demanded by these patients.
\end{abstract}

KEY WORDS: Psychotic disorders/diagnosis. Psychotic disorders/psychology. Somatoform disorders/diagnosis. Somatoform disorders/psychology. Diagnosis, differential.

\title{
REFERÊNCIAS
}

1. Feldman MD, Hamilton JC, Deemer HN. Factitious disorder. In: Phillips KA, editor. Somatoform and factitious disorders. Washington, DC: American Psychiatric Publishing; 2001. p. 129-66. (Review of Psychiatry series, v. 20, no. 3).

2. Fleming $A$, Eisendrath $S$. Factitious disorders. In: Tasman A, Kay J, Lieberman JA, editors. Psychiatry, v. 2. 2nd ed. Chichester: John Wiley \& Sons; 2003. p.1444-51.

3. Kaplan HI, Sadock BJ, Grebb JA. Compêndio de psiquiatria. 7a ed. Porto Alegre: Artmed; 1997. p. 598602.

4. Leamon MH, Feldman MD, Scott CL. Transtornos factícios e simulação. In: Hales RE, Yudofsky SC, editores. Tratado de psiquiatria clínica. $4 a$ ed. Porto Alegre: Artmed; 2006. p. 657-71.

5. Maxmen JS, Ward NG. Essential psychopathology and its treatment. 2nd ed. New York: WW Norton \& Company; 1995. p. 304-309.
6. Oliveira JT. Aspectos comportamentais das síndromes de dor crônica. Arq Neuropsiquiatr. 2000;58(2-A):3605.

7. Plassmann R. Munchausen syndromes and factitious diseases. Psychother Psychosom. 1994;62(1-2):61726.

8. Plewes JM, Fagan JG. Trastornos facticios y simulación. In: Hales RE, Yudofsky SC, Talbott JA, editors. Tratado de psiquiatría. 2a ed. Barcelona: Ancora; 1996. p. 659-68

9. Pope HG, Jonas JM, Jones B. Factitious psychosis: phenomenology, family history, and long-term outcome of nine patients. Am J Psychiatry. 1982;139(11):14803.

10. Suresh TR, Srinivasan TN. Claimed simulation of insanity - a coping strategy in mania. Br J Psychiatry. 1990;157:603-5.

11. Yates WR. Other psychiatric syndromes: adjustment disorder, factitious disorder, illicit steroid abuse. In: Winokur G, Clayton PJ. The medical basis of psychiatry. 2nd ed. Philadelphia: WB Saunders; 1994. p. 273-85. 